\title{
Aggressive primary urethral malignancy masquerading as stricture urethra: Unusual presentation of a rare malignancy
}

\author{
Sridhar Parnandi ${ }^{1}$, Ravindra G Varma ${ }^{2}$, Amit Saple ${ }^{2}$ \\ From ${ }^{1}$ Consultant Urologist, ${ }^{2}$ Senior Consultant Urologist, Department of Urology, Asian Institute of Nephrology and Urology, Visakhapatnam, \\ Andhra Pradesh, India
}

\begin{abstract}
Bladder outlet obstruction in an elderly male patient is most of the time diagnosed as prostatomegaly or stricture urethra unless there is a high index of suspicion for other differential diagnoses. Rare tumors like urethral malignancy may be misdiagnosed due to their varied and delayed mode of presentation. Here, we present a case of a patient who presented with acute urinary retention and was suspected to be a stricture urethra which ultimately turned out to be an aggressive urethral tumor. Investigations such as retrograde urethrogram, ultrasound, or cystoscopy which are the most common diagnostic modalities in a patient with lower urinary tract symptoms may not diagnose urethral tumors. Delayed diagnosis is common in these tumors resulting in a lesser chance for curative treatment. A high index of suspicion with a lesser threshold for investigations like MRI is pivotal for early diagnosis in these patients.
\end{abstract}

Key words: Bladder outlet obstruction, Stricture urethra, Retrograde urethrogram, Urethral malignancy

$\mathrm{U}$ rethral tumors are rare accounting for $1 \%$ of urologic malignancies [1]. Due to the rarity of these tumors, there are no established criteria for the diagnosis and the management protocol has not changed much over the years [2]. The mode of presentation can be varied and due to their aggressive nature, early diagnosis and treatment are the keys to successful tumor control.

Here, we present a case of bulbar urethral tumor which was masquerading as a case of stricture urethra due to its mode of presentation and rapidly progressed to metastatic disease. The rationale for presenting this case is due to the rarity of the disease and misdiagnosis which may happen due to its varied mode of presentation.

\section{CASE REPORT}

A 59-year-old male patient presented with a history of lower abdominal pain and retention of urine for 1 day. He had a history of obstructive voiding symptoms for 3 days. Foley's catheterization was attempted at an outside hospital but could not be passed.

On general examination, tachycardia was present but blood pressure was normal. The patient was afebrile. On local examination, the bladder was palpable till umbilicus. External

\section{Access this article online}

Received - 06 September 2021

Initial Review - 22 September 2021

Accepted - 02 October 2021

DOI: $10.32677 /$ ijcr.v7i10.3089 urethral meatus was normal. Digital rectal examination revealed Grade 1 prostatomegaly. There was mid induration in the perineum but no visible swelling. Ultrasound showed a Grade 1 prostate of $30 \mathrm{cc}$ with normal echotexture and an overdistended bladder. With the suspicion of urethral rupture/urethral stricture, cystoscopy and guided catheterization were planned.

Cystoscopy revealed normal anterior urethra till bulbar urethra where narrowing was present with mucosal edema and irregularity (Fig. 1). The cystoscope was not passable but the ureteroscope could be passed with resistance into the bladder. Guidewire was placed but Foleys could not be negotiated over it. Diagnosis of stricture urethra was made and suprapubic cystostomy (SPC) was done. The patient was discharged and advised to review after 2-3 weeks for further workup.

After 3 weeks, retrograde urethrogram (RGU) was done which showed contrast extravasation in the bulbar urethra with the irregular posterior urethra (Fig. 2). A magnetic resonance imaging (MRI) scan was planned in view of non-healing of the urethra after SPC and induration in the perineum which increased in size in three weeks. MRI revealed an ill-defined, heterogenous mass lesion involving the corpus spongiosum of the bulbar urethra, extending into the membranous and prostatic urethral region, these findings suggestive of urethral malignancy (Fig. 3a). Contrast-enhanced computed tomography (CECT) chest and abdomen were done for metastatic workup which

Correspondence to: Sridhar Parnandi, Department of Urology, Asian Institute of Nephrology and Urology, Visakhapatnam, Andhra Pradesh, India. E-mail: parnandi.sridhar@gmail.com

(C) 2021 Creative Commons Attribution-NonCommercial 4.0 International License (CC BY-NC-ND 4.0). 


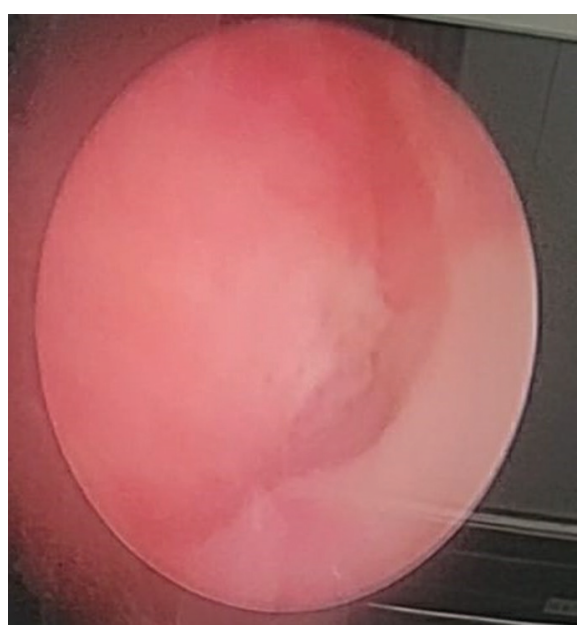

Figure 1: Cystoscopy image of the patient

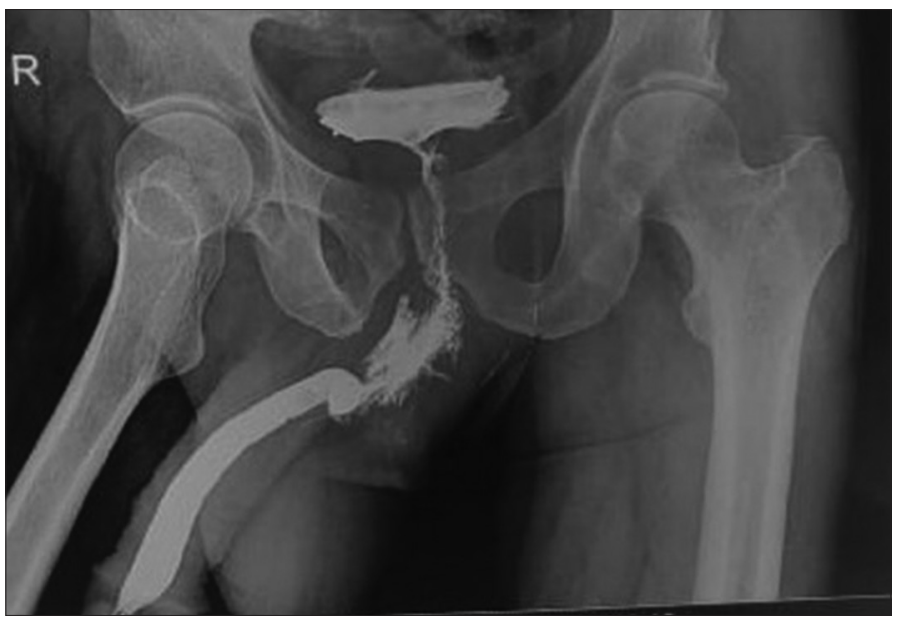

Figure 2: Retrograde urethrogram showing contrast extravasation in the bulbar urethra with irregular posterior urethra
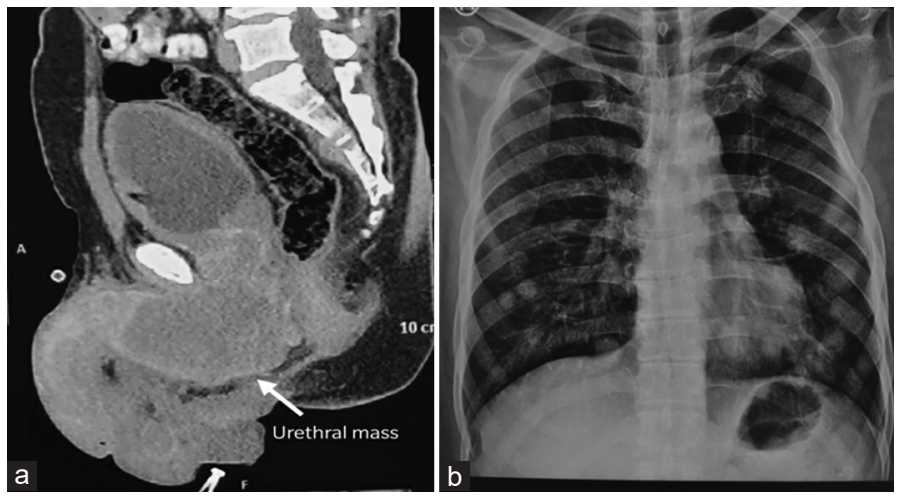

Figure 3: (a) MRI image and (b) chest X-ray of the patient

revealed multiple lung metastases and a lytic bone lesion in the sacrum. X-ray chest showed multiple cannonball opacities in both lungs (Fig. 3b). Cystoscopy and biopsy from the urethra were attempted but could not be taken due to severe narrowing in the bulbar urethra. A trucut biopsy was done from the indurated lesion in the perineum which revealed the diagnosis to be poorly differentiated squamous cell carcinoma. The patient was referred to an oncologist for palliative chemotherapy but succumbed to the disease within 1 month even before starting chemotherapy.

\section{DISCUSSION}

Stricture urethra and prostatomegaly are the most common diagnoses in a patient presenting with urinary retention or bladder outlet obstruction. Urethral tumors present usually with lower urinary tract symptoms (LUTS), hematuria, or dysuria. Being an uncommon diagnosis in a patient with LUTS or urinary retention, urethral malignancy is rarely suspected in these patients. Ultrasound, RGU, or cystoscopy which are common diagnostic modalities used for patients with LUTS, may not be useful for the primary diagnosis of urethral malignancy.

Our patient was an elderly male presenting with urinary retention and a palpable bladder with a failed catheterization attempt. An initial diagnosis of prostatomegaly or urethral stricture was naturally made and SPC was done to relieve the obstruction. The induration in the perineum which was insignificant at the initial clinical examination rapidly increased in size within three weeks. Being a rare diagnosis there tends to be more threshold for doing an MRI in these patients and hence diagnosis may be significantly delayed. The literature search revealed only a few similar case reports of urethral malignancy presenting with retention of urine. Young-Joo Kim [3] reported two cases of female urethral malignancy presenting with retention of urine. Di Gregorio [4] reported a case of penile adenocarcinoma which proved to be an aggressive disease similar to our case where despite multimodal surgical and chemoradiotherapy, the patient rapidly succumbed to the disease. Badhiwala et al. [5] reported a rare case of urethral sarcoma which was treated for 2 years as a case of urethral stricture before being diagnosed as a metastatic urethral sarcoma. Being aggressive malignancies, urethral carcinomas rapidly metastasize. Early diagnosis is pivotal as a surgical intervention with complete tumor excision is the only curative intervention for these patients.

Etiological factors for urethral malignancy include chronic stricture, urethritis, or human papillomavirus 16 infection [6]. Penile tissue contains several types of cells, and different histological types of penile malignancies can develop. Bulbomembranous urethra is the most common site for urethral malignancy and squamous cell carcinoma is the most common histological subtype [7]. Transitional cell carcinoma may be seen in $90 \%$ of prostatic urethral malignancy and $10 \%$ of bulbomembranous urethral tumors [8]. Metastatic lesions to the penis are also not uncommon with primary tumors in the bladder, prostate, rectum-sigmoid, kidney, hematologic system, lung, testis, etc. Anterior urethral tumors present relatively early and are more easily managed surgically compared to posterior urethral tumors which are most of the time locally advanced or metastatic at the time of presentation [9]. Tumors of the anterior urethra spread to inguinal nodes, whereas, posterior urethral tumors spread to pelvic nodes. Tissue diagnosis of a urethral lesion can be done by various methods. Distal lesions can simply be excised. Proximal tumors can be biopsied cystoscopically with cold-cup biopsy forceps or through transurethral/percutaneous routes. Urothelial tumors of the prostatic urethra can be biopsied with a resectoscope loop. 
The surgical approach to primary urethral cancer of the bulbar urethra is determined by tumor stage. Transurethral resection or excision with end-to-end anastomosis can be done for low stage lesions. Radical surgery which includes total penectomy and urethrectomy with possible cystoprostatectomy and pelvic lymphadenectomy is required for complete tumor control in higher stage lesions. Pathologic lymph node metastasis is a poor prognostic factor. Single modality therapy has poorer results compared to multimodal treatment. Multimodal therapy with cisplatin-based chemotherapy, radiation, and consolidation surgery has greatly improved the overall prognosis and recurrence rates [10].

\section{CONCLUSION}

In a patient presenting with the lower urinary tract symptoms or bladder outlet obstruction, urethral malignancy should be kept in mind as a differential diagnosis especially in elderly patients. Bulbar urethral tumors may present as a mass in the perineum, hence a thorough physical examination, a high index of suspicion, and a less threshold for investigations like MRI is pivotal for early diagnosis and management of these patients.

\section{REFERENCES}

1. Eng TY, Naguib M, Galang T, Fuller CD. Retrospective study of the treatment of urethral cancer. Am J Clin Oncol 2003;26:558-62.
2. Dalbagni G, Zhang ZF, Lacombe L, Herr HW. Male urethral carcinoma: Analysis of treatment outcome. Urology 1999;53:1126-32.

3. Kim YJ, Huh JS. Female urethral adenocarcinoma: An unusual cause of acute urinary retention. Korean J Urol 2008;49:759-61.

4. Di Gregorio M, Nollevaux MC, Dupont M, Lorge F, D'Hondt L. A rare case of urinary retention in an adult due to a primary penile Tumor. Hematol Med Oncol 2020;5:1-5.

5. Badhiwala N, Chan R, Zhou HJ, Shen S, Coburn M. Sarcomatoid carcinoma of male urethra with bone and lung metastases presenting as urethral stricture. Case Rep Urol 2013;2013:931893.

6. Wiener JS, Liu ET, Walther PJ. Oncogenic human papillomavirus Type 16 is associated with squamous cell cancer of the male urethra. Cancer Res 1992;52:5018-23.

7. Mostofi FK, Davis CJ Jr., Sesterhenn IA. Carcinoma of the male and female urethra. Urol Clin North Am 1992;19:347-58.

8. Grigsby PW, Herr HW. Urethral tumors. In: Comprehensive Textbook of Genitourinary Oncology. $2^{\text {nd }}$ ed. Philadelphia, PA: Lippincott Williams and Wilkins; 2000. p. 1133-40.

9. Zeidman EJ, Desmond P, Thompson IM. Surgical treatment of carcinoma of the male urethra. Urol Clin North Am 1992;19:359-72.

10. Zinman LN, Vanni AJ. Management of proximal primary urethral cancer: Should multidisciplinary therapy be the gold standard? Urol Clin North Am 2016;43:505-13.

Funding: None; Conflicts of Interest: None Stated.

How to cite this article: Parnandi S, Varma RG, Saple A. Aggressive primary urethral malignancy masquerading as stricture urethra: Unusual presentation of a rare malignancy. Indian J Case Reports. 2021;7(10):449-451. 